\title{
Rapid Quantification of Chlorpromazine Residues in Pork Using Nanosphere-Based Time-Resolved Fluorescence Immunoassay Analyzer
}

\author{
Wei Wang $\mathbb{D}^{1},{ }^{1}$ Jingneng Wang, $^{2}$ Min Wang, ${ }^{1}$ and Juan Shen ${ }^{1}$ \\ ${ }^{1}$ Key Laboratory of Meat Processing and Quality Control, MOE, Key Laboratory of Meat Processing, \\ MARA, Jiangsu Synergetic Innovation Center of Meat Processing and Quality Control, Nanjing Agricultural University, \\ No. 1 Weigang, Nanjing, Jiangsu 210095, China \\ ${ }^{2}$ Shanghai Xiongtu Biotechnology Co., Ltd., No. 21 Guangfulin Road, Shanghai 201600, China \\ Correspondence should be addressed to Wei Wang; wangwei821220@njau.edu.cn
}

Received 9 December 2020; Revised 25 February 2021; Accepted 2 March 2021; Published 9 March 2021

Academic Editor: Danilo Corradini

Copyright (c) 2021 Wei Wang et al. This is an open access article distributed under the Creative Commons Attribution License, which permits unrestricted use, distribution, and reproduction in any medium, provided the original work is properly cited.

\begin{abstract}
Immunochromatographic assays are good analytical tools for the detection of drug residues. We report a nanosphere-based timeresolved fluorescence immunoassay (nano-TRFIA) based on a monoclonal antibody and a portable TRFIA analyzer for the rapid quantification of chlorpromazine (CPZ) residues in pork. Under optimal conditions, the nano-TRFIA detected CPZ residues within 6 min of sample pretreatment. The results showed good linearity $\left(R^{2}=0.991\right)$, with a limit of detection (LOD) of $0.32 \mu \mathrm{g} / \mathrm{kg}$, a wide dynamic range of $0.46-10.0 \mu \mathrm{g} / \mathrm{kg}$, and coefficients of variation (CVs) of the overall intrabatch and interbatch assays of $7.34 \%$ and $7.65 \%$, respectively. The nano-TRFIA was also used to detect CPZ at different spiked concentrations in pork, and the results were confirmed via ultraperformance liquid chromatography-tandem mass spectrometry (UPLC-MS/MS). The nanoTRFIA was evaluated for the analysis of six commercial pork samples, and the results agreed well with those obtained via UPLCMS/MS, without significant differences $(P>0.05)$. Therefore, the proposed nano-TRFIA is a powerful alternative for the rapid and accurate quantification of $\mathrm{CPZ}$ residues in pork to meet the required Chinese maximum residue limits for veterinary drugs in foods.
\end{abstract}

\section{Introduction}

Chlorpromazine $(\mathrm{CPZ})$ is a typical phenothiazine antipsychotic drug [1-3] and is common in clinical veterinary practice because of its strong sedative and antiemetic effects $[4,5]$. The addition of CPZ into animal feeds causes sedation, hypnosis, weight gain, and fattening and shortens the slaughter time. Chlorpromazine can also attenuate the stress response and maintenance needs of animals and can decrease their weight loss and mortality during long-distance transportation and thus prevent the reduction of meat quality $[6,7]$. However, CPZ residues in animal products can adversely affect human health [8-11]. As early as 1997, the European Union issued Commission Regulation (EC) No. 17/97 which banned the addition of CPZ into feeds. Japan has also stipulated that $\mathrm{CPZ}$ should not be present in foodstuffs of animal origin. According to the National Food Safety Standard on Maximum Residue Limits for Veterinary Drugs in Foods (GB31650-2019) in China, CPZ is allowed for treatment but should not be present in foods of animal origin. Nevertheless, some vendors still illegally add CPZ into edible animal feeds.

Detection methods for $\mathrm{CPZ}$ residues are underdeveloped compared with those for other antipsychotic drugs [12]. The CPZ residue detection methods can be classified into two types: the first type is based on chromatography, and it includes liquid microextraction-liquid chromatography [13], liquid chromatography-coulometry [14], high-performance liquid chromatography (HPLC) [15], liquid chromatography-mass spectrometry [16], and gas chromatography-mass spectrometry [17]. These methods require advanced laboratories, tedious sample pretreatment, 
long detection time, expensive apparatus, and operation by experienced personnel. Moreover, only a small number of samples can be analyzed at one time; therefore, these methods are not suitable for in situ detection or batch screening. The other type is based on immunological analyses, including enzyme-linked immunosorbent assay (ELISA), which is the most widely used. ELISA has been widely utilized to detect drug residues because of its high specificity, sensitivity, and simple operation [18-20]. However, this method suffers from low stability during quantification, low-temperature storage of reagents, short action time, long detection cycle, and unsuitability for testing a small number of samples. Thus, there is an urgent need for rapid, simple, convenient, and reliable detection methods.

Time-resolved fluorescence immunoassay (TRFIA) was developed in the early 1980s. This technology uses complexes of lanthanides and fluorescent markers and has been successfully applied to labeled immunoassays and clinical medicine $[21,22]$. However, the detection sensitivity needs to be amplified through fluorescence enhancement due to the low fluorescence intensities of lanthanide complexes. Nanosphere-based TRFIA (nano-TRFIA) is a novel TRFIA that combines the long-lived fluorescence of rare-earth elements $\left(\mathrm{Eu}^{3+}, \mathrm{Tb}^{3+}\right.$, etc. $)$ with the signal amplification effect of nanospheres. Rare-earth elements and their complexes are codoped into nanospheres. After surface activation, an antibody is coupled onto the surface of a label to form a complex. During the immunoassay, this complex can elevate the sensitivity and expand the linear dynamic range [23]. In addition to facile operation, stable tracing, wide quantification, application ranges, and no radioactive contamination, nano-TRFIA can also offer higher sensitivity and accuracy than conventional methods; this is because polystyrene nanospheres containing thousands of lanthanide chelates have been developed as attractive labels with remarkable signal amplification potential [24]. Thus, in this study, we established a nano-TRFIA for the rapid quantification of $\mathrm{CPZ}$ residues in pork under the optimal experimental conditions described in our previous study [25]. Our goal is to provide references for quality and safety monitoring and the supervision and formulation of detection standards.

\section{Materials and Methods}

2.1. Materials and Reagents. Chlorpromazine, promethazine (PMZ), thioridazine (TDZ), acepromazine (ACP), prochlorperazine (PCZ), haloperidol (HAL), and fluphenazine (FPZ) were purchased from Dr. Ehrenstorfer Co. (Augsburg, Germany). Horseradish peroxidase-labeled goat anti-mouse immunoglobulin G (IgG) and tetramethylbenzidine (TMB) and stop buffer were obtained from Cell Signaling Technology (St. Louis, USA). Chlorpromazine-bovine serum albumin and mouse anti-CPZ monoclonal antibody were prepared in our laboratory [26]. Goat anti-mouse IgG was provided by Beijing Dingguo Changsheng Biotechnology Co., Ltd. (Beijing, China). Bovine serum albumin (BSA), 1-ethy1-3-(3-dimethylaminopropyl) hydrochloride (EDC), tert-butyl methyl ether (TBME), and MES buffer (0.05 M, pH 6.0) were purchased from SigmaAldrich (St. Louis, MO, USA). Europium-chelate-coated nanospheres (PS-COOH) with a size of $300 \mathrm{~nm}$ were synthesized and provided by Bangs Laboratories, Inc. (Fishers, IN, USA). Millipore 135 nitrocellulose (NC) membrane with a flow rate of $135 \mathrm{~s} / 4 \mathrm{~cm}$, conjugate pad, sample pad, and absorbent pad were obtained from Millipore (MA, USA). All solutions were prepared in deionized water $(18.2 \mathrm{M} \Omega \cdot \mathrm{cm}$, an Arium ${ }^{\circledR}$ Pro ultrapure water purification system from Sartorius, Göttingen, Germany). Two CPZ-positive pork samples labeled A and B were obtained from the Supervision, Inspection and Testing Center for Quality of Meat Products (Nanjing). Four pork samples labeled C, D, E, and $F$ were acquired from a local supermarket in Nanjing, China. Other analytically pure reagents were obtained from Nanjing Chemical Reagent Co., Ltd. (Nanjing, China).

2.2. Detection of Cross-Reactivity of Monoclonal Antibody. The specificity of the antibody was determined via indirect competitive ELISA. Chlorpromazine, PMZ, TDZ, ACP, PCZ, HAL, and FPZ solutions at a series of concentrations were prepared and coated on 96-well microplates (Corning Inc., Corning, NY, USA). Mouse anti-CPZ monoclonal antibody was used as the primary antibody, and $0.01 \mathrm{M}$ phosphate-buffered saline (PBS, $\mathrm{pH} 7.2$ ) and CPZ were used as the negative and positive controls, respectively. Horseradish peroxidase-labeled goat anti-mouse IgG was used as the secondary antibody. After the antibody was incubated at room temperature for $1 \mathrm{~h}$, washed, and mixed with TMB for color development, the optical density (OD) at $450 \mathrm{~nm}$ was measured using a Synergy H1 microplate reader (Bio-Tek, Winooski, VT, USA). The cross-reactivity (CR) was calculated as follows:

$$
\mathrm{CR}(\%)=\frac{\mathrm{IC}_{50}(\mathrm{CPZ})}{\mathrm{IC}_{50}(\mathrm{CPZ} \text { structural analog })} \times 100 \%,
$$

where $\mathrm{IC}_{50}$ is the half-maximal inhibitory concentration.

2.3. TRFIA Apparatus. The XT8201A portable TRFIA analyzer was codeveloped with Shanghai Xiongtu Biotechnology Co., Ltd., China, and used for the quantitative determination of CPZ. A light-emitting diode lamp served as the excitation source at $365 \mathrm{~nm}$, and signals were acquired at $610 \mathrm{~nm}$ using a photomultiplier tube (PMT). The fluorescence signal was recorded using the PMT after a $400 \mu$ s delay when the exciting light was irradiated on the test strip area. Meanwhile, the fluorescence with a short relaxation time decayed rapidly, reducing the background noise and achieving a high signal-to-noise ratio. The fluorescence signals on the test line ( $\mathrm{T}$ line) and the control line ( $\mathrm{C}$ line) peaks were processed using data processing software for quantitative analysis.

2.4. Labeling of Mouse Anti-CPZ Monoclonal Antibody by Fluorescent Nanospheres. Europium fluorescent nanospheres $(100 \mu \mathrm{L})$ were added to $500 \mu \mathrm{L}$ of $0.05 \mathrm{M}$ boric acid 
buffer ( $\mathrm{pH}$ 8.0). The mixture was vortexed and added to $100 \mu \mathrm{L}$ of $10 \mathrm{M}$ EDC solution (prepared with $0.05 \mathrm{M}$ MES buffer, $\mathrm{pH}$ 6.0). The obtained mixture was activated by shaking at room temperature for $30 \mathrm{~min}$ and centrifuged at $8000 \mathrm{r} / \mathrm{min}$ for $10 \mathrm{~min}$ to discard the supernatant. The precipitate was then redissolved with $500 \mu \mathrm{L}$ of $0.05 \mathrm{M}$ boric acid buffer ( $\mathrm{pH} 8.0$ ), ultrasonicated for $5 \mathrm{~min}$, and added to $100 \mu \mathrm{L}$ of mouse anti-CPZ monoclonal antibody. After the amount of fluorescent nanosphere-conjugated antibody was adjusted to $30 \mu \mathrm{g} / \mathrm{mL}$, the reaction was performed at room temperature for $2 \mathrm{~h}$. Subsequently, $50 \mu \mathrm{L}$ of $0.05 \mathrm{M}$ boric acid buffer ( $\mathrm{pH}$ 8.0) containing 10\% BSA was added, and the mixture was reacted at room temperature for $2 \mathrm{~h}$ on a thermomixer and centrifuged at $8000 \mathrm{r} / \mathrm{min}$ for $10 \mathrm{~min}$ to discard the supernatant. The precipitate was then redissolved with $500 \mu \mathrm{L}$ of $0.05 \mathrm{M}$ boric acid buffer $(\mathrm{pH}$ 8.0) and ultrasonicated for $5 \mathrm{~min}$. The labeled fluorescent nanospheres $(1: 800)$ were sprayed onto a bonding pad with a T2DDA film-spotting, gold-spraying instrument (Shanghai Hangan Electronic Technology Co., Ltd., Shanghai, China). After drying, the nanospheres were sealed and stored at room temperature prior to use.

2.5. Assembly of Nano-TRFIA Kit. The assembly process of the nano-TRFIA kit is illustrated in Figure 1 . First, $1.0 \mathrm{mg} / \mathrm{mL}$ CPZ-BSA and $1.0 \mathrm{mg} / \mathrm{mL}$ goat anti-mouse IgG were sprayed onto an NC membrane with a T2DDA film-spotting, goldspraying instrument (Shanghai Hangan Electronic Technology Co., Ltd., Shanghai, China) as a $\mathrm{T}$ line and a $\mathrm{C}$ line, respectively (distance: about $1.0 \mathrm{~cm}$ ), and dried overnight at $37^{\circ} \mathrm{C}$. Afterward, the sample pad, conjugate pad, NC membrane, and absorbent pad were sequentially pasted on a polyvinyl chloride (PVC) board. The conjugate pad and sample pad were pasted at the $\mathrm{T}$ line. The conjugate pad and NC membrane overlapped by about $1-2 \mathrm{~mm}$. The absorbent pad was pasted at the $C$ line, and the pad overlapped with the NC membrane by about 1-2 mm. The PVC board was then cut into a $3.55 \mathrm{~mm}$ wide strip with a C6 strip-cutting machine (Shanghai Hangan Electronic Technology Co., Ltd., China) and placed into a plastic card to fabricate a nano-TRFIA kit and stored at room temperature for further experiments.

2.6. Sample Pretreatment for Nano-TRFIA. Pork tissue was homogenized at 10,000 r/min for $1 \mathrm{~min}$, and $2.00 \mathrm{~g}$ (accurate to $0.01 \mathrm{~g}$ ) was put into a $50 \mathrm{~mL}$ centrifuge tube with $500 \mu \mathrm{L}$ of $5 \mathrm{M} \mathrm{NaOH}$ solution and vortexed for $30 \mathrm{~s}$. The sample was then mixed with $200 \mu \mathrm{L}$ of acetonitrile, vortexed for $80 \mathrm{~s}$, and then shaken thoroughly for another $30 \mathrm{~min}$. Next, $12 \mathrm{~mL}$ of TBME was added, vortexed for $80 \mathrm{~s}$, and centrifuged at $4^{\circ} \mathrm{C}$ and $13,000 \mathrm{r} / \mathrm{min}$ for $15 \mathrm{~min}$. Subsequently, the supernatant was blow-dried with nitrogen at $40^{\circ} \mathrm{C}$, and the residue was redissolved with $2 \mathrm{~mL}$ of methanol. The solution was diluted with $0.01 \mathrm{M}$ PBS ( $\mathrm{pH}$ 7.2) and used for immediate measurement.

2.7. Nano-TRFIA for CPZ Detection. All operations were performed at room temperature $\left(22^{\circ} \mathrm{C}-28^{\circ} \mathrm{C}\right)$. First, $100 \mu \mathrm{L}$ of the extracted solutions described above was added into a sample well of the nano-TRFIA kit. After incubation at $37^{\circ} \mathrm{C}$ for $6 \mathrm{~min}$, the nano-TRFIA kit was immediately inserted into the test strip slot of a portable TRFIA analyzer, and the fluorescence signals on the $\mathrm{T}$ line and $\mathrm{C}$ line were directly recorded under ultraviolet light (Figure 2).

\subsection{Nano-TRFIA Standard Curve and Determination of} Linearity. A CPZ standard sample was dissolved in methanol, calibrated, and mixed with a $1.0 \mathrm{~g} / \mathrm{kg}$ standard solution. The system was then diluted to $10.0,5.0,2.0,1.0,0.5,0.25$, $0.125,0.0625$, and $0 \mu \mathrm{g} / \mathrm{kg}$ solutions with $0.01 \mathrm{M}$ PBS ( $\mathrm{pH}$ 7.2). The CPZ standard solution was tested using the nanoTRFIA kit, and a portable TRFIA analyzer (Shanghai Xiongtu Biotechnology Co., Ltd., China) was used to record the fluorescence intensities of $\mathrm{T}$ and $\mathrm{C}$ lines and their ratios. Each standard was tested five times to plot the standard curve. The curve was drawn with $\operatorname{LnX}$ as the $x$-axis (where $X$ is the concentration of competitive antigen), and the $B / B_{0}$ ratio of the standard solution is seen at each concentration as the $y$-axis. The linear range was determined, where $B$ is the $T / C$ ratio after the addition of the $\mathrm{CPZ}$ standard solution and $B_{0}$ is the ratio in the presence of $0 \mu \mathrm{g} / \mathrm{kg}$ standard solution.

2.9. Determination of Limit of Detection and Limit of Quantification of Nano-TRFIA. Twenty blank samples were randomly taken for the nano-TRFIA strip test to calculate the mass concentration of the blank sample. The average $(X)$ and standard deviation (SD) were obtained. The limit of detection (LOD) and limit of quantification (LOQ) were calculated according to $\mathrm{LOD}=X+3 \mathrm{SD}$ and $\mathrm{LOQ}=X+10 \mathrm{SD}$, respectively.

2.10. Detection of Accuracy and Reproducibility of NanoTRFIA. The accuracy and reproducibility of this method were represented by intrabatch and interbatch coefficients of variation (CVs). Chlorpromazine standard solutions at three different concentrations were tested. Two batches of the same sample were tested, and each batch was detected 10 times. The average of three CVs for each batch was employed as the intrabatch $\mathrm{CV}$, and the average of two intrabatch CVs was used as the overall $\mathrm{CV}$. The interbatch $\mathrm{CV}$ was calculated by measuring the CVs of two batches at each concentration 20 times and then averaging the values.

2.11. Comparison of Nano-TRFIA and Ultraperformance Liquid Chromatography-Tandem Mass Spectrometry Results. To validate the nano-TRFIA results, six negative minced pork samples were treated with $\mathrm{CPZ}$ standard solutions at $1.0,2.0,4.0,6.0,8.0$, and $10.0 \mu \mathrm{g} / \mathrm{kg}$ and then subjected to ultraperformance liquid chromatography-tandem mass spectrometry (UPLC-MS/MS) (Waters Corp., Milford, MA, USA) $[16,27]$. The spiked samples were pretreated with acetonitrile to precipitate proteins, followed by extraction with TBME. Separation was performed on a Waters ACQUITY UPLC BEH C18 column $(50 \mathrm{~mm} \times 2.1 \mathrm{~mm}$, $1.7 \mu \mathrm{m})$. The column temperature was $30^{\circ} \mathrm{C}$, and the sample 


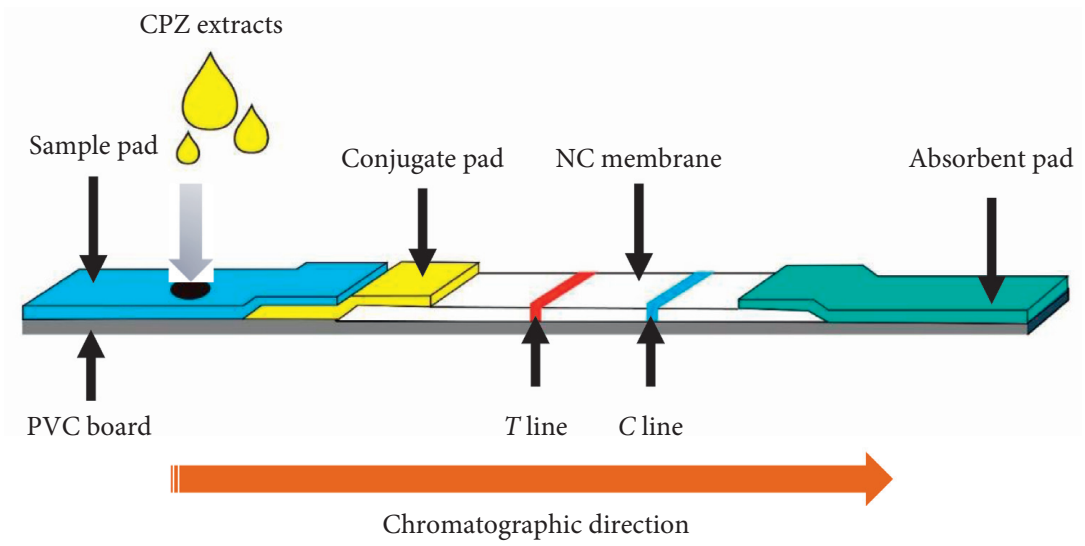

FIGURE 1: Description of the principle of nanosphere-based time-resolved fluorescence immunoassay (nano-TRFIA). NC: nitrocellulose; PVC: polyvinyl chloride; CPZ: chlorpromazine.

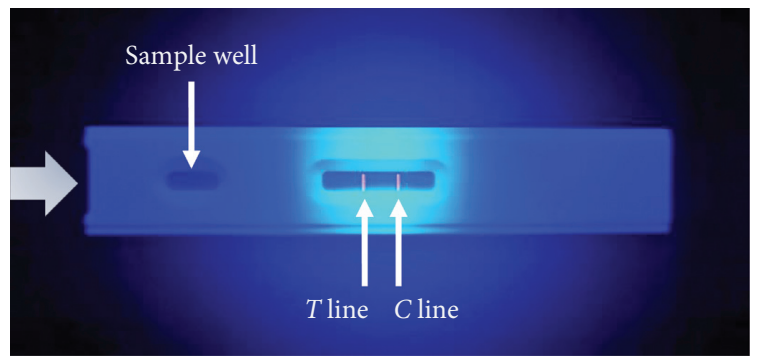

FIGURE 2: Image of chlorpromazine detection via nanospherebased time-resolved fluorescence immunoassay under ultraviolet light.

injection volume was $10.0 \mu \mathrm{L}$. Gradient elution was performed with solutions A (acetonitrile) and B $(0.1 \%(v / v)$ formic acid in water) as the mobile phases, at a flow rate of $0.3 \mathrm{~mL} / \mathrm{min}$ : $0-2 \mathrm{~min}, 10 \% \mathrm{~A}$ and $90 \% \mathrm{~B} ; 2-4 \mathrm{~min}, 60 \% \mathrm{~A}$ and $40 \% \mathrm{~B}$; and $4-5 \mathrm{~min}, 10 \% \mathrm{~A}$ and $90 \% \mathrm{~B}$ [25]. Detection was performed via positive-ion electrospray ionization in multiple reaction monitoring mode. The transition of $\mathrm{m} / z$ 319.27-85.96 was used to quantify CPZ. The recovery rate and relative standard deviation (RSD) of the samples were calculated.

2.12. Application in Real Pork Sample. To validate our strategy, six pork samples from different products were used to detect CPZ. Chlorpromazine from pork samples was extracted as described, and the extracts were analyzed through both nano-TRFIA and UPLC-MS/MS.

\section{Results and Discussion}

3.1. CR of Monoclonal Antibody. Indirect competitive ELISA was conducted to detect the CRs between mouse anti-CPZ monoclonal antibody and other six structurally relevant compounds. As presented in Table 1, the antibody had an excellent specificity (CR: $<0.77 \%)$, with mild reactivity with only PCZ (CR: 5.74\%) among the considered chemicals. Therefore, there was either low or no CR during CPZ detection, which ensures the specificity and accuracy of the
TABLE 1: CR of mouse anti-CPZ monoclonal antibody.

\begin{tabular}{lcc}
\hline Standard & $\mathrm{IC}_{50}(\mu \mathrm{g} / \mathrm{kg})$ & $\mathrm{CR}(\%)$ \\
\hline CPZ & 15.38 & 100 \\
PCZ & 267.90 & 5.74 \\
ACP & $>2000$ & $<0.77$ \\
TDZ & $>2000$ & $<0.77$ \\
PMZ & $>2000$ & $<0.77$ \\
HAL & $>2000$ & $<0.77$ \\
FPZ & $>2000$ & $<0.77$ \\
\hline
\end{tabular}

CR, cross-reactivity; ACP, acepromazine; CPZ, chlorpromazine; FPZ, fluphenazine; HAL, haloperidol; $\mathrm{IC}_{50}$, half-maximal inhibitory concentration; PCZ, prochlorperazine; PMZ, promethazine; TDZ, thioridazine.

proposed nano-TRFIA. We postulate that the stronger binding of the antibody to PCZ than to the other chemicals is because the key structure of PCZ is similar to that of CPZ.

3.2. Standard Curve and Linearity of Nano-TRFIA. To establish a standard curve, the fluorescence intensities on the $\mathrm{T}$ line and the $\mathrm{C}$ line with different $\mathrm{CPZ}$ concentrations were recorded using a portable TRFIA analyzer (Figure 3). As shown in Figure 4, the standard curve was plotted with the logarithm of the concentration of the competitive antigen $\mathrm{CPZ}$ standard as the $x$-axis and the $B / B_{0}$ ratio as the $y$-axis. The curve shows good linearity when the logarithm ranges from -2.773 to 2.303 , corresponding to concentrations of 0.0625 to $10.0 \mu \mathrm{g} / \mathrm{kg}$. The fitted linear regression equation is $Y=-0.135 X+0.547\left(R^{2}=0.991\right)$, meeting the requirement for a linear relationship.

3.3. LOD and LOQ of Nano-TRFIA. The $B / B_{0}$ values measured with 20 blank samples were substituted into the above linear regression equation, giving an average $\mathrm{CPZ}$ concentration of $0.26 \mu \mathrm{g} / \mathrm{kg}$ and an SD of 0.02 . According to the equations $\mathrm{LOD}=\bar{X}+3 \mathrm{SD}$ and $\mathrm{LOQ}=\bar{X}+10 \mathrm{SD}$, the LOD and LOQ were calculated as $0.32 \mu \mathrm{g} / \mathrm{kg}$ and $0.46 \mu \mathrm{g} / \mathrm{kg}$, respectively. The established nano-TRFIA thus had a much higher sensitivity than those previously reported $[7,17,26,28,29]$. This suggests that the nano-TRFIA has practical application value. 


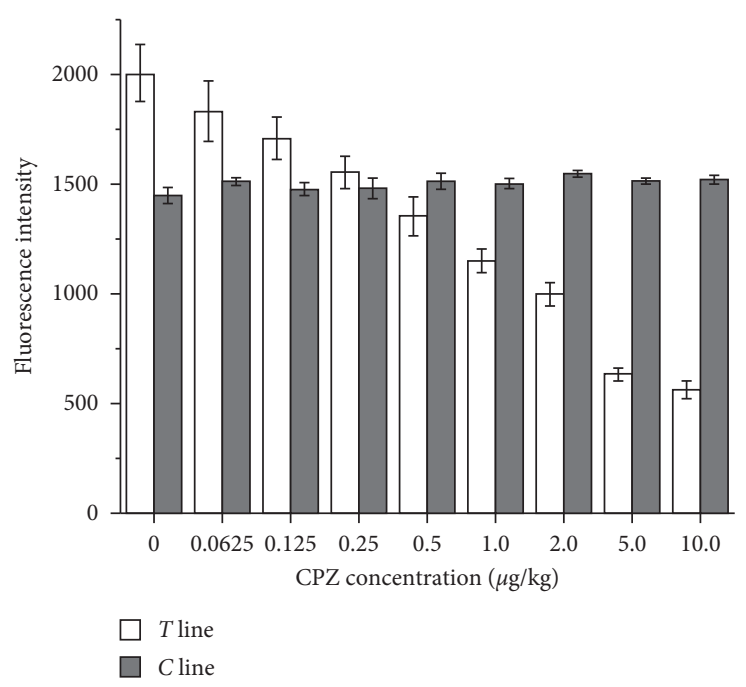

FIGURE 3: Fluorescence intensity of nanosphere-based time-resolved fluorescence immunoassay with $0,0.0625,0.125,0.25,0.5,1.0,2.0,5.0$, and $10.0 \mu \mathrm{g} / \mathrm{kg}$ of chlorpromazine (CPZ). The $x$-axis is the fluorescence intensity on the T line and C line peaks at different CPZ concentrations; the $y$-axis is a standard solution at different concentrations.

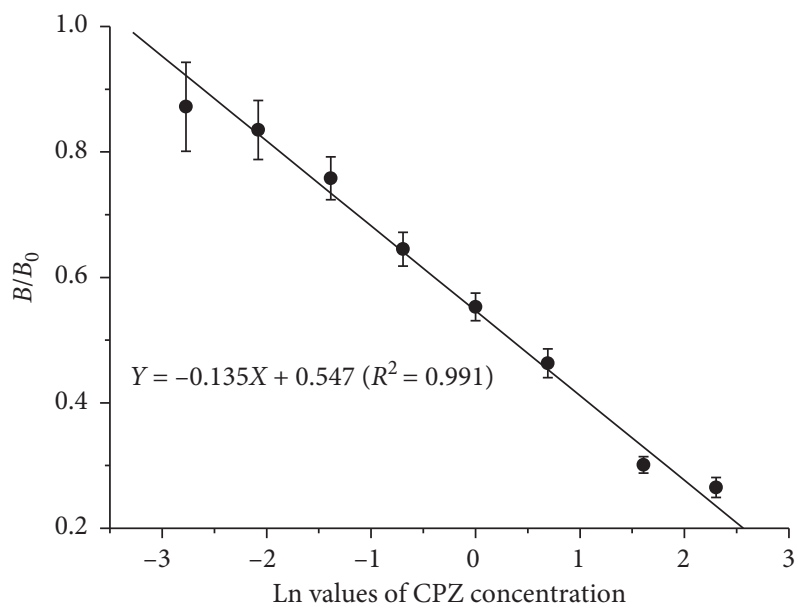

FIGURE 4: Standard curve of chlorpromazine (CPZ) detection via nanosphere-based time-resolved fluorescence immunoassay (eight levels of serial dilution containing $0.0625,0.125,0.25,0.5,1.0,2.0,5.0$, and $10.0 \mu \mathrm{g} / \mathrm{kg}$ of CPZ were prepared in PBS). The $x$-axis is the Ln value of $\mathrm{CPZ}$ concentration; the $y$-axis is the $B / B_{0}$ ratio of standard solution at each concentration.

3.4. Accuracy and Reproducibility of Nano-TRFIA. The accuracy of the nano-TRFIA was determined by setting three concentrations of CPZ standard solutions (1.0, 5.0, and $10.0 \mu \mathrm{g} / \mathrm{kg}$ ), dividing the samples into two batches, and repeatedly testing each batch 10 times (Table 2). The intrabatch CVs of the first and second batches were $6.32 \%$ and $8.36 \%$, respectively. The overall intrabatch and interbatch CVs were $7.34 \%$ and $7.65 \%$, respectively. This method is highly accurate and repeatable since all CVs were lower than $10.0 \%$.

3.5. Validation by UPLC-MS/MS. To verify the accuracy of this method, the correlation of the measurement results with those of UPLC-MS/MS was analyzed. For UPLC-MS/MS, the standard curve was plotted via CPZ standard solutions at mass concentrations of $0.25,0.5,1.0,2.5,5.0$, and $10.0 \mu \mathrm{g} / \mathrm{kg}$, with a regression equation of $Y=11028.80 X-1403.87$
$\left(R^{2}=0.994\right)$. The retention time of $\mathrm{CPZ}$ was $2.2 \mathrm{~min}$. The LOD was $0.25 \mu \mathrm{g} / \mathrm{kg}$, and the LOQ was $1.0 \mu \mathrm{g} / \mathrm{kg}$.

Samples were tested via nano-TRFIA and UPLC-MS/MS under the same spiked concentration (Table 3). The results were subjected to linear regression analysis, giving an equation of $Y=0.957 X+0.147\left(R^{2}=0.999\right)$ (Figure 5). The $\mathrm{CPZ}$ in the samples could be accurately and reliably detected via the established nano-TRFIA.

3.6. Determination and Evaluation of Real Pork Samples. To examine the applicability of the established nano-TRFIA, we selected six different pork samples and compared the results with those of nano-TRFIA and UPLC-MS/MS (Table 4). The results obtained using the nano-TRFIA kits were nearly consistent with the UPLC-MS/MS detection results. There was no significant difference between the results of the 
TABLE 2: CPZ detection by nano-TRFIA.

\begin{tabular}{|c|c|c|c|c|c|c|}
\hline \multirow{2}{*}{$\mathrm{CPZ}$ concentration $(\mu \mathrm{g} / \mathrm{kg})$} & \multicolumn{3}{|c|}{ First batch $(n=10)$} & \multicolumn{3}{|c|}{ Second batch $(n=10)$} \\
\hline & Mean value $(\mu \mathrm{g} / \mathrm{kg})$ & $\mathrm{SD}$ & CV (\%) & Mean value $(\mu \mathrm{g} / \mathrm{kg})$ & $\mathrm{SD}$ & CV (\%) \\
\hline 1.0 & 0.97 & 0.02 & 2.61 & 0.95 & 0.06 & 8.51 \\
\hline 5.0 & 4.86 & 0.04 & 7.82 & 5.04 & 0.04 & 8.85 \\
\hline 10.0 & 11.87 & 0.03 & 8.53 & 11.31 & 0.02 & 7.71 \\
\hline
\end{tabular}

$\mathrm{CPZ}$, chlorpromazine; CV, coefficient of variation; nano-TRFIA, nanosphere-based time-resolved fluorescence immunoassay; SD, standard deviation.

TABLE 3: Comparison of nano-TRFIA and UPLC-MS/MS for CPZ detection in pork $(n=5)$.

\begin{tabular}{|c|c|c|c|c|c|c|}
\hline \multirow[b]{2}{*}{ Spiked concentration $(\mu \mathrm{g} / \mathrm{kg})$} & \multicolumn{3}{|c|}{ UPLC-MS/MS } & \multicolumn{3}{|c|}{ Nano-TRFIA } \\
\hline & $\begin{array}{c}\text { Detected concentration } \\
(\mu \mathrm{g} / \mathrm{kg})\end{array}$ & Recovery (\%) & RSD (\%) & $\begin{array}{c}\text { Detected concentration } \\
(\mu \mathrm{g} / \mathrm{kg})\end{array}$ & Recovery (\%) & RSD (\%) \\
\hline 1.0 & $0.96 \pm 0.03$ & 96.40 & 3.99 & $0.93 \pm 0.05$ & 92.60 & 5.59 \\
\hline 2.0 & $1.92 \pm 0.04$ & 96.20 & 2.25 & $1.92 \pm 0.04$ & 95.80 & 2.11 \\
\hline 4.0 & $3.92 \pm 0.09$ & 98.00 & 2.63 & $3.89 \pm 0.12$ & 97.15 & 3.59 \\
\hline 6.0 & $5.98 \pm 0.10$ & 99.73 & 1.80 & $6.04 \pm 0.13$ & 100.63 & 2.40 \\
\hline 8.0 & $8.03 \pm 0.16$ & 100.35 & 2.23 & $8.09 \pm 0.15$ & 101.15 & 2.09 \\
\hline 10.0 & $10.06 \pm 0.14$ & 100.58 & 1.56 & $10.47 \pm 0.45$ & 104.70 & 4.77 \\
\hline
\end{tabular}

CPZ, chlorpromazine; nano-TRFIA, nanosphere-based time-resolved fluorescence immunoassay; RSD, relative standard deviation; UPLC-MS/MS, ultraperformance liquid chromatography-tandem mass spectrometry.

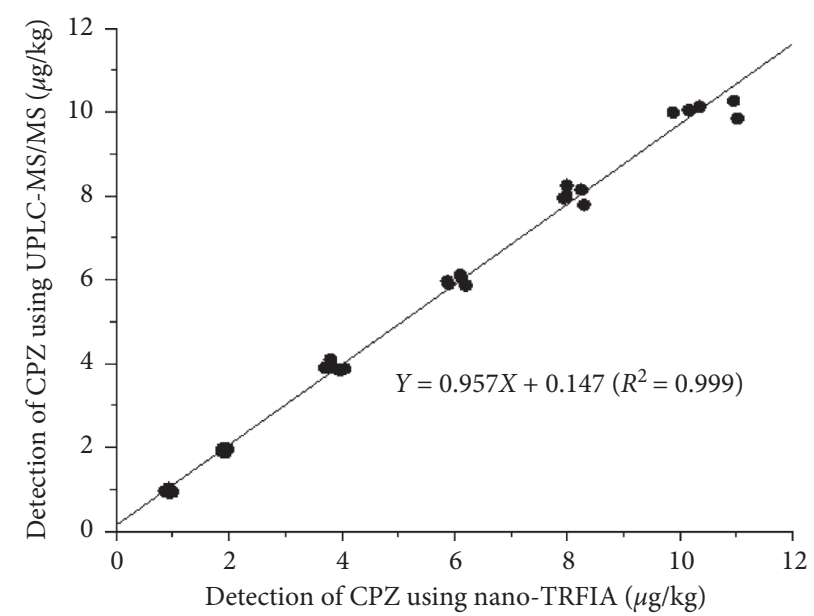

FIGURE 5: Correlation analysis of nanosphere-based time-resolved fluorescence immunoassay (nano-TRFIA) and ultraperformance liquid chromatography-tandem mass spectrometry (UPLC-MS/MS) results (six negative minced pork samples were added to chlorpromazine (CPZ) standard solutions at $1.0,2.0,4.0,6.0,8.0$, and $10.0 \mu \mathrm{g} / \mathrm{kg}$ ). The $x$-axis is the detection result of CPZ using nano-TRFIA; the $y$-axis is the detection result of $\mathrm{CPZ}$ using UPLC-MS/MS.

TABLE 4: Comparison of nano-TRFIA and UPLC-MS/MS for CPZ detection in real pork samples $(n=5)$.

\begin{tabular}{lcc}
\hline Sample & & CPZ found $(\mu \mathrm{g} / \mathrm{kg})$ \\
\hline A & UPLC-MS/MS & Nano-TRFIA \\
B & $1.62 \pm 0.07$ & $1.57 \pm 0.06$ \\
C & $1.95 \pm 0.09$ & $1.92 \pm 0.05$ \\
D & $\mathrm{Nd}$ & $\mathrm{Nd}$ \\
E & $\mathrm{Nd}$ & $\mathrm{Nd}$ \\
F & $\mathrm{Nd}$ & $\mathrm{Nd}$ \\
\hline
\end{tabular}

CPZ, chlorpromazine; nano-TRFIA, nanosphere-based time-resolved fluorescence immunoassay; UPLC-MS/MS, ultraperformance liquid chromatographytandem mass spectrometry; nd, not detected. 
two methods $(P>0.05)$. The data could be used to evaluate the applicability and reliability of the newly developed method. Thus, this nano-TRFIA is a potential alternative to chromatography for the regulatory analysis of $\mathrm{CPZ}$ residues in foodstuffs of animal origin.

\section{Conclusion}

Detecting CPZ residues in meat is important; hence, several countries have formulated standards for $\mathrm{CPZ}$ residues. Although China has continuously focused on the monitoring of CPZ during food safety inspections and has enforced a strict ban on the addition of CPZ to animal feed, $\mathrm{CPZ}$ residues have still been detected in pork in recent years. The strategy reported in this study combines nano-TRFIA with immunochromatography to realize high specificity, sensitivity, reproducibility, and a wide linear range. The detection results meet the requirements for quantifying actual samples. This method is readily operable and does not require expensive instrumentation. Moreover, the approach can quickly measure CPZ residues in foodstuffs of animal origin in situ and may have application value for other drug residues.

\section{Data Availability}

The original data used to support the findings of this study can be obtained from the corresponding author upon request.

\section{Conflicts of Interest}

The authors declare that there are no conflicts of interest regarding the publication of this paper.

\section{Acknowledgments}

The authors express their gratitude to Mr. Xudong Zhu for his assistance in the research and development of the nanoTRFIA kit. This research was financially supported by the Natural Science Foundation of Jiangsu Province (no. BK20191307) and the National Key R\&D Program of China (no. 2018YFC1602804).

\section{References}

[1] B. Thornley, C. E. Adams, and A. G. Awad, "Chlorpromazine versus placebo for the treatment of schizophrenia: a symstematic review," Schizophrenia Research, vol. 29, no. 1-2, p. 155, 1998.

[2] C. A. W. Rijcken, T. B. M. Monster, J. R. B. J. Brouwers, and L. T. W. de Jong-van den Berg, "Chlorpromazine equivalents versus defined daily doses: how to compare antipsychotic drug doses?" Journal of Clinical Psychopharmacology, vol. 23, no. 6, pp. 657-659, 2003.

[3] J. Cooper, P. Delahaut, T. L. Fodey, and C. T. Elliott, "Development of a rapid screening test for veterinary sedatives and the beta-blocker carazolol in porcine kidney by ELISA," The Analyst, vol. 129, no. 2, pp. 169-174, 2004.
[4] S. Song, X. Shi, R. Li et al., "Extraction of chlorpromazine with a new molecularly imprinted polymer from pig urine," Process Biochemistry, vol. 43, no. 11, pp. 1209-1214, 2008.

[5] J. J. Liu and S. M. Yang, "Development of detect of chlorpromazine residue," China Animal Husbandry \& Veterinary Medicine, vol. 35, no. 10, pp. 141-144, 2008.

[6] L. Amaral, M. Viveiros, and J. Molnar, "Antimicrobial activity of phenothiazines,” In Vivo, vol. 18, no. 6, pp. 725-731, 2004.

[7] P. Lu, Z. N. Qu, W. Q. Tan, R. Zheng, and J. Jiang, "Studies on the GC-MS analysis of chlorpromazine residue in pig liver," Chinese Journal of Animal Quarantine, vol. 23, no. 7, pp. 30-31, 2006.

[8] A. H. Abadi, S. Rafatullah, and A. A. Al-Badr, "Chlorpromazine," in Analytical Profiles of Drug Substances and Excipients, H. G. Brittain, Ed., pp. 97-165, Academic Press, San Diego, CA, USA, 1999.

[9] Y. Ito, S. Sato, M. Son et al., "Bioelectric toxicity caused by chlorpromazine in human lung epithelial cells," Toxicology and Applied Pharmacology, vol. 183, no. 3, pp. 198-206, 2002.

[10] R. Frötschl, S. Weickardt, S. Staszewski, G. Kaufmann, and P. Kasper, "Effects of chlorpromazine with and without UV irradiation on gene expression of HepG2 cells," Mutation Research, vol. 575, no. 1-2, pp. 47-60, 2005.

[11] T. Li and Q. X. Zhou, "Ecotoxicological effect of chlorpromazine and its influence on human health," Chinese Journal of Ecology, vol. 25, no. 12, pp. 1554-1558, 2006.

[12] R. Feng, Q. Liang, and R. T. Zhan, "Optimization of national standard method for determination of chlorpromazine residues in pork," Medical Information, vol. 28, no. 9, p. 124, 2015.

[13] M. Cruz-Vera, R. Lucena, S. Cárdenas, and M. Valcárcel, "Determination of phenothiazine derivatives in human urine by using ionic liquid-based dynamic liquid-phase microextraction coupled with liquid chromatography," Journal of Chromatography B: Analytical Technologies in the Biomedical and Life Sciences, vol. 877, no. 1-2, pp. 37-42, 2009.

[14] M. A. Saracino, M. Amore, E. Baioni, C. Petio, and M. A. Raggi, "Determination of selected phenothiazines in human plasma by solid-phase extraction and liquid chromatography with coulometric detection," Analytica Chimica Acta, vol. 624, no. 2, pp. 308-316, 2008.

[15] A. Asghari, E. Fahimi, M. Bazregar, M. Rajabi, and L. Boutorabi, "Rapid determination of some psychotropic drugs in complex matrices by tandem dispersive liquid-liquid microextraction followed by high performance liquid chromatography," Journal of Chromatography B: Analytical Technologies in the Biomedical and Life Sciences, vol. 1052, pp. 51-59, 2017.

[16] L. Zhang, W. Huang, and X. H. Jiang, "Determination of chlorpromazine in porcine muscle using high performance liquid chromatography-tandem mass spectrometry," Journal of Chinese Pharmaceutical Sciences, vol. 24, no. 10, pp. 690694, 2015.

[17] M. N. Shan, X. F. Xu, Y. X. Pu et al., "Determination of chlorpromazine in pork by gas chromatography-mass spectrometry," Chinese Journal of Food Hygiene, vol. 25, no. 5, pp. $438-440,2013$.

[18] S. X. Lu, Y. L. Zhang, J. T. Liu et al., "Preparation of antipefloxacin antibody and development of an indirect competitive enzyme-linked immunosorbent assay for detection of pefloxacin residue in chicken liver," Journal of Agricultural and Food Chemistry, vol. 54, no. 19, pp. 6995-7000, 2006.

[19] C. B. Zhao, W. Liu, H. L. Ling et al., "Preparation of antigatifloxacin antibody and development of an indirect competitive enzyme-linked immunosorbent assay for the 
detection of gatifloxacin residue in milk," Journal of Agricultural and Food Chemistry, vol. 55, no. 17, pp. 6879-6884, 2007.

[20] Z. Q. Liu, S. X. Lu, C. H. Zhao et al., "Preparation of antidanofloxacin antibody and development of an indirect competitive enzyme-linked immunosorbent assay for detection of danofloxacin residue in chicken liver," Journal of the Science of Food and Agriculture, vol. 89, no. 7, pp. 1115-1121, 2009.

[21] J.-C. G. Bünzli and C. Piguet, "Taking advantage of luminescent lanthanide ions," Chemical Society Reviews, vol. 34, no. 12, pp. 1048-1077, 2005.

[22] J. Z. Shen, Z. Zhang, Y. Yao et al., "A monoclonal antibodybased time-resolved fluoroimmunoassay for chloramphenicol in shrimp and chicken muscle," Analytica Chimica Acta, vol. 575, no. 2, pp. 262-266, 2006.

[23] D. Wang, Z. Zhang, P. Li et al., "Europium nanospheres-based time-resolved fluorescence for rapid and ultrasensitive determination of total aflatoxin in feed," Journal of Agricultural and Food Chemistry, vol. 63, no. 47, pp. 10313-10318, 2015.

[24] Y. Shen, S. Xu, and D. He, "A novel europium chelate coated nanosphere for time-resolved fluorescence immunoassay," PLoS One, vol. 10, no. 6, Article ID e0129689, 2015.

[25] M. Wang, "Establishment and evaluation of rapid quantitative detection of chlorpromazine in animal-derived foods by timeresolved fluorescence immunoassay," Master's thesis, Nanjing Agricultural University, Nanjing, China, 2018.

[26] M. Wang, W. Wang, Q. Q. Lü et al., "Rapid detection of chlorpromazine in pork tissue by indirect competitive ELISA," Journal of Nanjing Agricultural University, vol. 43, no. 1, pp. 172-177, 2020.

[27] B. P. Li, J. Hua, H. Li et al., "Determination of chlorpromazine illegally added in 3 kinds of veterinary preparations by UPLCMS/MS," Chinese Journal of Veterinary Drug, vol. 49, no. 5, pp. 32-36, 2015.

[28] General Administration of Quality Supervision, Inspection and Quarantine of the People's Republic of China (AQSIQ), Standardization Administration of the People's Republic of China (SAC), National Standard of the People's Republic of China, Method for Determination of Acetopromaizine, Chlorpromazine, Haloperidol, Propionylpromazine, Xylazine, Azaperone, Azaperol and Carazolol Residues in Porcine Kidney and Muscle Tissues-LC-MS-MS Method, GB/T 20763-2006, General Administration of Quality Supervision, Beijing, China, 2006.

[29] Y. L. Hong, X. F. Hao, and K. Dong, "HPLC determination of chlorpromazine residue in animal-derived foods," Food Science, vol. 30, no. 14, pp. 269-271, 2009. 